\title{
Correction to: Wrong way maps in uniformly finite homology and homology of groups
}

\author{
Alexander Engel ${ }^{1}$ \\ Received: 22 August 2019 / Accepted: 30 August 2019 / Published online: 21 September 2019 \\ (c) Tbilisi Centre for Mathematical Sciences 2019

\section{Correction to: J. Homotopy Relat. Struct. (2018) 13:423-441 https://doi.org/10.1007/s40062-017-0187-x}

There is an error in the proof of Theorem 2.16 of [2]. It occured at the end of the second-to-last paragraph of the proof: there it is claimed that “...the application of $\theta$ to it vanishes..." which needs in general an additional assumption like triviality of the normal bundle. The main theorem of the paper now reads as stated below (under the first set of assumptions in its statement one can circumvent the second-to-last paragraph in the proof completely). The arXiv-version [1] was already updated accordingly.

Main Theorem Let $M$ be a closed and connected manifold and let $N \hookrightarrow M$ be a closed, connected submanifold of codimension $q \geq 1$ such that the inclusion induces an injective map on fundamental groups $\pi_{1}(N) \hookrightarrow \pi_{1}(M)$. Assume further one of the following:

1. That the normal bundle $v$ of the embedding $N \hookrightarrow M$ is oriented (for some fixed coefficient group) and, in the case $q \geq 2$, that $\pi_{i}(M)=0$ for $2 \leq i \leq q$.

2. That the normal bundle $v$ of the embedding $N \hookrightarrow M$ is trivial.

Further, in the case $q=2$ that $\pi_{2}(N) \rightarrow \pi_{2}(M)$ is surjective, and in the case $q>2$ that $\pi_{i}(M)=0$ for $2 \leq i \leq q-1$ and that $\pi_{q}(N) \rightarrow \pi_{q}(M)$ is surjective.

Communicated by Thomas Schick.

The original article can be found online at https://doi.org/10.1007/s40062-017-0187-x.

$\triangle \quad$ Alexander Engel

alexander.engel@mathematik.uni-regensburg.de

1 University of Regensburg, Regensburg, Germany 
Then we construct a homomorphism $H_{*}\left(B \pi_{1} M\right) \rightarrow H_{*-q}\left(B \pi_{1} N\right)$ such that the diagram

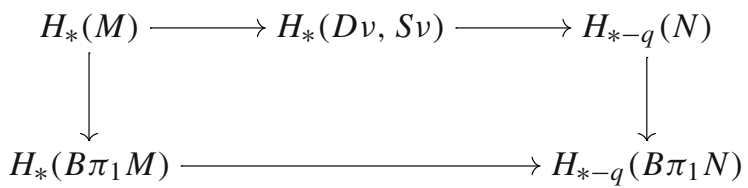

commutes, where the map $H_{*}(M) \rightarrow H_{*}(D v, S v)$ is the Thom-Pontryagin collapse, the map $H_{*}(D v, S v) \rightarrow H_{*-q}(N)$ is cap-product with the Thom class of $v$, and the vertical maps are induced by the classifying maps.

\section{References}

1. Engel, A.: Wrong way maps in uniformly finite homology and homology of groups. arXiv:1602.03374 (2016)

2. Engel, A.: Wrong way maps in uniformly finite homology and homology of groups. J. Homotopy Relat. Struct. 13(2), 423-441 (2018)

Publisher's Note Springer Nature remains neutral with regard to jurisdictional claims in published maps and institutional affiliations. 